\title{
A Hybrid Algorithm for Spatial and Wavelet Domain Image Restoration
}

\author{
You-Wei Wen ${ }^{a, b}$, Wai-Ki Ching ${ }^{b}$, Michael K. $\mathrm{Ng}^{b}$ and Hong $\mathrm{Liu}^{c}$ \\ ${ }^{a}$ Faculty of Applied Mathematics, Guangdong University of Technology, Guangzhou, China; \\ ${ }^{b}$ Department of Mathematics, The University of Hong Kong, Pokfulam Road, Hong Kong; \\ ${ }^{c}$ Institute of Geology and Geophysics, Chinese Academy of Sciences Beijing, China.
}

\begin{abstract}
The recent algorithm ForWaRD based on the two steps: (i) the Fourier domain deblurring and (ii) wavelet domain denoising, shows better restoration results than those using traditional image restoration methods. In this paper, we study other deblurring schemes in ForWaRD and demonstrate such two-step approach is effective for image restoration.
\end{abstract}

Keywords: Restoration, Constrained Least Squares, Adaptive Regularized Least Squares, Wavelet

\section{INTRODUCTION}

Image restoration problem is an important image processing task in many real world applications. The observed image results from blurring operations performed on the original image, and it is also corrupted by additive noises. The blurring of images often occurs from a relative motion between the camera and the original scene, from defocusing of the lens system, or from atmospheric turbulence.

In digital image processing, the discrete imaging model of the degradation process can be represented by using vectors and matrices. With the lexicographical ordering of the original image $\mathbf{f}$ and the observed image $\mathbf{g}$, their relationship can be expressed as follows:

$$
\mathrm{g}=\mathbf{H f}+\mathbf{n}
$$

Here $\mathbf{H}$ is the blurring matrix and $\mathbf{n}$ is a vector of zero-mean Gaussian white noise with variance $\sigma^{2}$. The task of image restoration is to recover the original image $\mathbf{f}$ from the observed image $\mathbf{g}$.

Image restoration is an ill-posed problem. The simple approach of performing the inverse transformation is not feasible. The inverse transformation may be singular or may be very ill-conditioned, a small perturbation in the observed image can produce a large perturbation in the restored image. Regularization theory is often used to handle such ill-conditioned problems. Image restoration methods are usually based on the construction of a regularized energy functional. The energy functional is the weighted sum of the two terms. The first term is the data fitting term $\|\mathbf{g}-\mathbf{H f}\|_{2}^{2}$ and the second term is the stabilized energy functional which contains some prior information about the original image $\mathbf{f}$ to alleviate the problem of ill-conditioning characteristics. By adjusting the regularization (weighting) parameter, a compromise is achieved to suppress the noise and preserve the nature of the original image. We remark that deblurring and denoising are performed simultaneously in the restoration process. In the literature, Constrained Least Squares (CLS) and Adaptive Regularized Least Squares (ARLS) are two common image restoration methods, see for instance. ${ }^{1}$

Further author information: (Send correspondence to Y. Wen.)

Y.Wen: E-mail: wenyouwei@yahoo.com, The research of this author is supported in part by the grant of the project of oil resources in pre-cenozoic layer in Gulf of Bohai No. KZCX1SW-18, and Guangdong Natural Science Foundation of Grant No. 032475.

W.Ching: E-mail: wkc@maths.hku.hk, The research of this author is supported in part by Hong Kong Research Grants Council Grant No. HKU 7126/02P and HKU CRCG Grant Nos. 10204436, 10205105.

M.Ng: E-mail: mng@maths.hku.hk. The research of this author is supported in part by Hong Kong Research Grants Council Grant Nos. HKU 7130/02P, 7046/03P and 7035/04P.

Visual Communications and Image Processing 2005, edited by Shipeng Li,

Fernando Pereira, Heung-Yeung Shum, Andrew G. Tescher, Proc. of SPIE Vol. 5960

(SPIE, Bellingham, WA, 2005) $\cdot 0277-786 X / 05 / \$ 15 \cdot$ doi: 10.1117/12.633379

Proc. of SPIE Vol. 5960 59605V-1 
The use of wavelet in image restoration is a relatively prevalent concept. Its success is due to the fact that signals and images usually have sparse wavelet representations. Therefore a few but significant wavelet coefficients can be used in the representation. Banham and Katsaggelos ${ }^{2}$ have developed and studied a multi-scale Kalman filter in a wavelet domain for image processing applications. Recently, Figueiredo ${ }^{3}$ proposed and developed an $\mathrm{EM}$ algorithm for image restoration in a wavelet domain. A review on different wavelet-based image restoration methods can also be found in Figueiredo and Nowak's paper. ${ }^{3}$

The use of the two-step approach for the task of image restoration is recently studied by several researchers. Nowak and $\mathrm{Thul}^{4}$ first proposed the two-step approach when they studied the linear shift invariant inverse problem arising from photon-limited imaging. The first step is to take the inverse of the convolution kernel directly. The second step is to perform denoising in the wavelet domain. Such two-step method is further developed and studied by Neelamani et al. ${ }^{5}$ and it is called ForWaRD. Both theoretical analysis of ForWaRD and simulation results show that this two-step method performs very well for image restoration. The main aim of this paper is to further consider other deblurring schemes in ForWaRD, and present experimental results to demonstrate that the two-step approach is indeed effective for image restoration.

The outline of this paper is as follows. In Section 2, we review on ForWaRD. In Section 3, we present other deblurring schemes. In Section 4, experimental results are given to illustrate the proposed method. Finally, we give concluding remarks in Section 5.

\section{A REVIEW ON FORWARD IMAGE RESTORATION}

\subsection{The Wiener Filter Deblurring.}

Under the periodic boundary condition, ${ }^{6}$ the blurring model in (1) can be re-written in the Fourier domain as follows: $\mathcal{G}=\mathcal{H} \mathcal{F}+\mathcal{N}$, where $\mathcal{G}, \mathcal{H}$ and $\mathcal{F}$ are the discrete Fourier transforms of $\mathbf{g}, \mathbf{H}$ and $\mathbf{f}$ respectively. The Wiener filter is the Mean Squares Error (MSE) optimal stationary linear filter for deblurring and denoising degraded images. In the Fourier domain, it can be expressed as follows:

$$
K=\frac{\mathcal{H}^{*} P_{x}}{|\mathcal{H}|^{2} P_{x}+\sigma^{2}}
$$

Here $\sigma^{2}$ is the noise variance and $P_{x}$ denotes the power spectra density of $\mathbf{f}$. Hillery and Chin proposed a method to estimate the power spectral denisity $P_{x} \cdot{ }^{7}$ When the original image is considered to be deterministic, ${ }^{7}$ $P_{x}=|\mathcal{F}|^{2}$. Thus the Fourier coefficients of the restored image are given by

$$
\widetilde{\mathcal{F}}=K \mathcal{G}=\frac{\mathcal{H}^{*}|\mathcal{F}|^{2} \mathcal{G}}{|\mathcal{H}|^{2}|\mathcal{F}|^{2}+\sigma^{2}}
$$

where $*$ denotes the conjugate transpose.

\subsection{The Wavelet-domain Denoising.}

When the blurring operator is scale homogeneous, Donoho ${ }^{8}$ proposed a very efficient threshold procedure for denoising which was called wavelet vaguelette denoising. If an image is corrupted by noises, then the noisy pixel values will be converted to noisy wavelet coefficients. The idea of this method is based on that large wavelet coefficients carry significant information and should be kept or shrunk; small coefficients are mostly noisy signal and can be ignored. Wavelet-based noise reduction algorithms are asymptotically near optimal for a wide class of signals corrupted by additive Gaussian white noises. However, such algorithms also work well when the noise is neither white noise nor Gaussian. ${ }^{9}$

Consider $\mathbf{g}=\mathbf{f}+\mathbf{n}$ and assume that the length of $\mathbf{g}$ is $2^{k}$. Denote the wavelet coefficients of $\mathbf{g}, \mathbf{f}$ and $\mathbf{n}$ by $\mathrm{g}^{\mathbf{w}}, \mathbf{f}^{\mathbf{w}}$ and $\mathbf{n}^{\mathbf{w}}$ respectively. ${ }^{10}$ Thus we obtain $\mathbf{g}^{\mathbf{w}}=\mathbf{f}^{\mathbf{w}}+\mathbf{n}^{\mathbf{w}}$ or $g_{i}^{w}=f_{i}^{w}+n_{i}^{w}$. Here $g_{i}^{w}, f_{i}^{w}$ and $n_{i}^{w}$ are the $i$ th wavelet coefficient of the observed signal, the original signal and the noise. In the soft-thresholding method, ${ }^{11}$ we replace $g_{i}^{w}$ by the shrunk coefficients $s_{\lambda}\left(g_{i}^{w}\right)$ where

$$
\bar{f}_{i}^{w}=s_{\lambda}\left(g_{i}^{w}\right)= \begin{cases}\operatorname{sgn}\left(g_{i}^{w}\right)\left(\left|g_{i}^{w}\right|-\lambda\right), & \left|g_{i}^{w}\right|>\lambda \\ 0, & \left|g_{i}^{w}\right| \leq \lambda\end{cases}
$$


While in the hard-thresholding method, we ignore all these coefficients $\left|g_{i}^{w}\right|$ which are be less than a threshold value $\lambda$. In this case, $s_{\lambda}\left(g_{i}^{w}\right)$ is given as follows:

$$
\bar{f}_{i}^{w}=s_{\lambda}\left(g_{i}^{w}\right)= \begin{cases}g_{i}^{w}, & \left|g_{i}^{w}\right|>\lambda \\ 0, & \left|g_{i}^{w}\right| \leq \lambda\end{cases}
$$

Donoho and Johnstone ${ }^{12}$ proposed the choice of parameter $\lambda=\sqrt{2 \ln 2^{k}} \sigma / 2$ and showed that the softthresholding is the $l_{2}$-optimal denoising filter in the wavelet domain. Ghael et al. ${ }^{13}$ proposed Wavelet-domain Wiener Filter (WWF) as the denoising scheme which can improve the MSE performance of the hard thresholding method. The wavelet coefficients of the restored image is defined by employing the Wiener filtering on each wavelet coefficient as follows:

$$
\tilde{f}_{i}^{w}=\frac{\left|f_{i}^{w}\right|^{2} g_{i}^{w}}{\left|f_{i}^{w}\right|^{2}+\sigma_{i}^{2}} .
$$

Here $\sigma_{i}^{2}$ denotes the noise variance at the $i$ th wavelet coefficient.

\subsection{ForWaRD.}

ForWaRD is a two-step method for image restoration problems. The algorithm is given as follows:

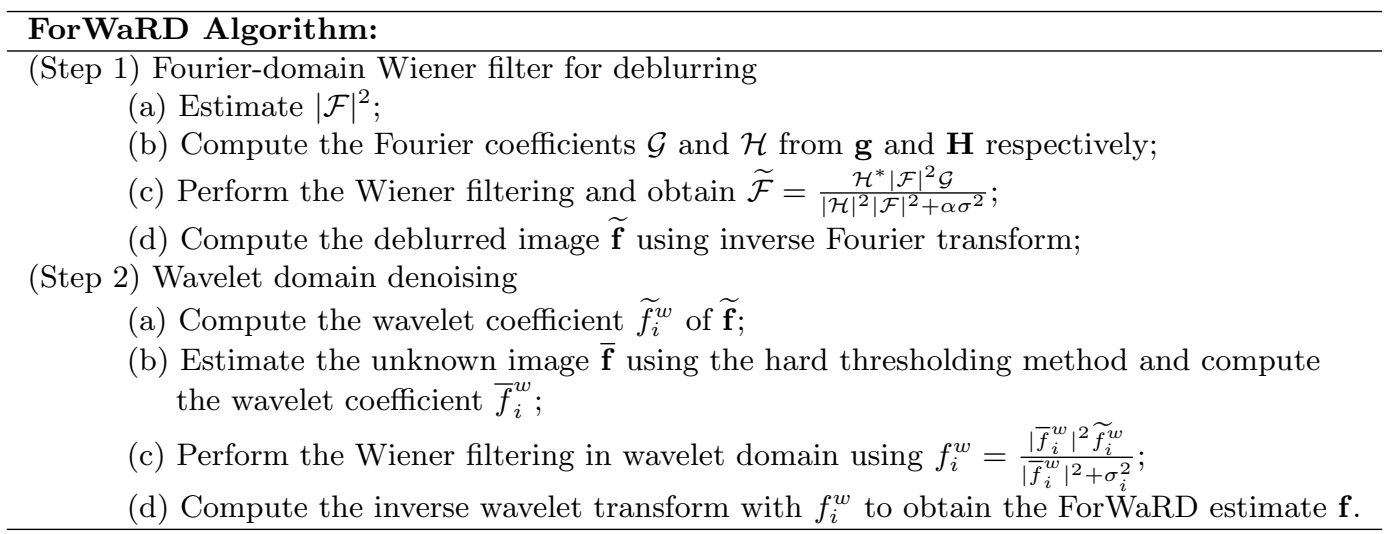

In (c) of Step 1, $\alpha$ is the weighting parameter introduced by Neelamani et al.. ${ }^{5}$ They also developed a software to employed ForWaRD, in their software, a flat signal spectrum is assumed, the coefficients in Step1(c) sets

$$
\widetilde{\mathcal{F}}=K \mathcal{G}=\frac{\mathcal{H}^{*} \mathcal{G}}{|\mathcal{H}|^{2}+\tau},
$$

\section{SPATIAL AND WAVELET DOMAIN RESTORATION}

Instead of the Wiener filter deblurring, other image deblurring schemes can be employed. Usually, they are formulated as the minimization of the following energy functional:

$$
\|\mathbf{g}-\mathbf{H f}\|_{\mathbf{2}}^{\mathbf{2}}+\operatorname{reg}(\mathbf{f}) .
$$

Here $\operatorname{reg}(\mathbf{f})$ is the regularization functional. ${ }^{1,14}$

\subsection{Constrained Least Squares (CLS) Restoration.}

The CLS restoration method was developed to determine $\widehat{\mathbf{f}}$ by solving the following minimization problem:

$$
\min _{\mathbf{f}}\|\mathbf{g}-\mathbf{H f}\|_{\mathbf{2}}^{\mathbf{2}}+\alpha\|\mathbf{R f}\|_{\mathbf{2}}^{\mathbf{2}} \text {. }
$$

Here $\alpha$ is called the regularization parameter and $\mathbf{R}$ is the regularization matrix. Usually, $\mathbf{R}$ is the identity matrix, in which a minimum norm on $\mathbf{f}$ subject to the noise norm equality constraint is sought, or $\mathbf{R}$ is the finite difference matrix, in which case the smoothness of the restored image is enhanced. The least squares solution of (8) is given by $\widehat{\mathbf{f}}=\left(\mathbf{H}^{T} \mathbf{H}+\alpha \mathbf{R}^{T} \mathbf{R}\right)^{-1} \mathbf{H}^{T} \mathbf{g}$, where $\mathbf{H}^{T}$ denotes the transpose of the matrix $\mathbf{H}$. 


\subsection{Adaptive Regularized Least Squares (ARLS) Restoration.}

Though the CLS solution can give a quite good quality restored image, the CLS restoration often introduces artifacts such as ringing effects near the sharp intensity transition. In ARLS method, several regularization parameters are employed in the restoration rather than a single parameter in the CLS method. The regularization functional of the ARLS method is defined as

$$
r e g(\mathbf{f})=\alpha\|\mathbf{R f}\|_{\mathbf{S}}^{\mathbf{2}}=\alpha \mathbf{f}^{\mathbf{T}} \mathbf{R}^{\mathbf{T}} \mathbf{S R f},
$$

where $\mathbf{S}$ is a diagonal matrix where the main diagonal entries $s_{i, i}$ are in $(0,1]$. The ARLS solution is given by $\widehat{\mathbf{f}}=\left(\mathbf{H}^{T} \mathbf{H}+\alpha \mathbf{R}^{T} \mathbf{S R}\right)^{-1} \mathbf{H}^{T} \mathbf{g}$. In general, $\mathbf{S}$ is defined such that the main diagonal entries $s_{i, i}$ are decreasing functions of the local variances of the original image $\mathbf{f}$. In the smooth regions, we should choose a large value $s_{i, i}$ in order to suppress the noise, while in the highly variation regions, a small value $s_{i, i}$ should be taken to deblur more significantly. ${ }^{1}$ The weighting coefficients in $S$ can be chosen on the basis of prior knowledge about the original image in order to minimize the error between the original image and the restored image. Biemond and Lagendijk proposed the following simple formula to calculate $s_{i, i}{ }^{15}$ :

$$
s_{i, i}=\left(\frac{\min _{i}\left\{\sigma_{i}^{2}\right\}}{\sigma_{i}^{2}}\right)^{\mu} .
$$

Here $\sigma_{i}^{2}$ denotes the local variance at the $i$-th pixel of the original image $\mathbf{f}$ and $\mu$ is a positive constant. Biemond and Lagendijk ${ }^{15}$ found that the value of $\mu$ can be set to 0.5 in practical applications. In our experimental results, we also employ this value to perform the spatial deblurring scheme in our proposed algorithm.

\subsection{The Hybrid Spatial and Wavelet Domain Algorithm.}

Our proposed algorithm is a two-step method for image restoration problems. The algorithm is given as follows:

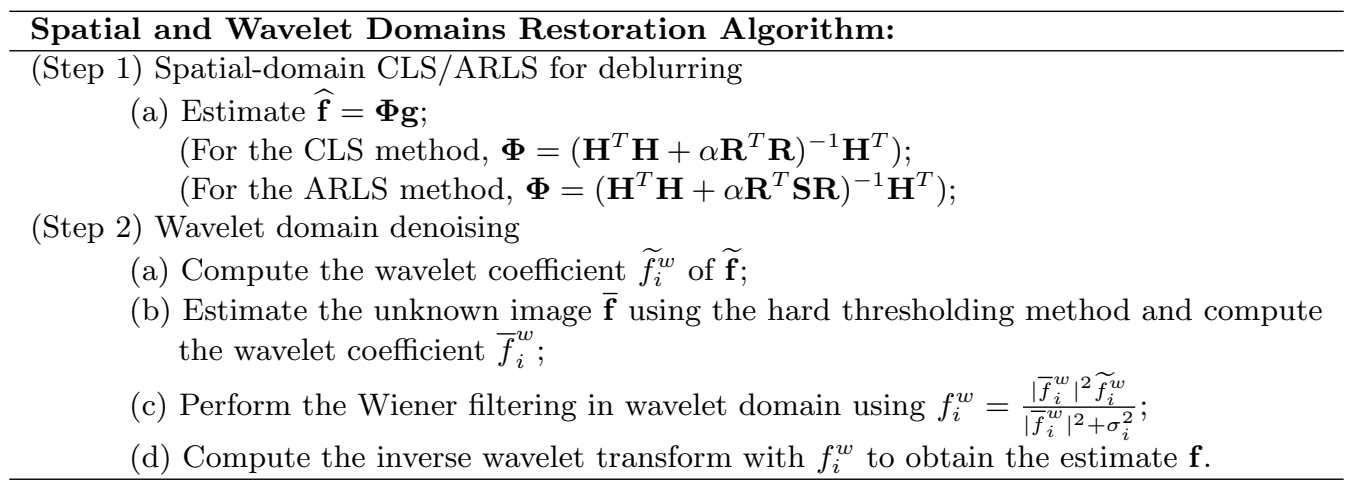

We remark when we apply the spatial domain ARLS scheme for deblurring, the residual noise becomes nonstationary. It is important to estimate the variance noise. Assume that complete knowledge of the original image is $\mathbf{f}$ known, then the noise is equal to the difference between the original image $\mathbf{f}$ and the estimated image $\widehat{\mathbf{f}}$, i.e., the residual noise $\widehat{\mathbf{n}}=\widehat{\mathbf{f}}-\mathbf{f}$. In practical,the original image $\mathbf{f}$ is unknown, we replace it by the pilot image $\mathbf{f}_{p}$ which is obtained by ARLS approach.

\section{SIMULATION RESULTS}

In this section, we illustrate the performance of our proposed spatial and wavelet domain image restoration algorithm. The Peak-Signal-to-Noise-Ratio (PSNR) is used to evaluate different methods. The PSNR is defined

by PSNR $=10 \log _{10} \frac{\|\mathbf{f}\|^{2}}{\|\mathbf{f}-\widetilde{\mathbf{f}}\|^{2}}$ where $\mathbf{f}$ and $\widetilde{\mathbf{f}}$ denote the original image and the restored image respectively, and the target image is $n$-by- $m$. Our codes are written in Matlab. The computational results by the Wiener filter and the ForWaRD were generated by using the software "For WaRD" which can be downloaded from 
http://www.dsp.rice.edu. In the software "ForWaRD", the deblurring scheme is used (6). In our test, the regularization matrix $\mathbf{R}$ is defined as:

$$
R=I_{n} \otimes\left(\begin{array}{ccccc}
2 & -1 & & & -1 \\
-1 & 2 & -1 & & \\
& \ddots & \ddots & \ddots & \\
& & -1 & 2 & -1 \\
-1 & & & -1 & 2
\end{array}\right)_{m}+\left(\begin{array}{ccccc}
2 & -1 & & & -1 \\
-1 & 2 & -1 & & \\
& \ddots & \ddots & \ddots & \\
& & -1 & 2 & -1 \\
-1 & & & -1 & 2
\end{array}\right)_{n} \otimes I_{m}
$$

We consider the blur and the noise variance tested by Banham and Katsaggelos ${ }^{2}$ and by Neelamani et $_{\text {al. }}{ }^{5}$ The $256 \times 256$ image was blurred by a $9 \times 9$-point box-car blur. The additive noise variance $\sigma^{2}$ was set such that the blurred SNR (BSNR) is $30 \mathrm{~dB}, 40 \mathrm{~dB}$ and $50 \mathrm{~dB}$ respectively. We compare different algorithms: the Wiener filter deblurring (Wiener); the ForWaRD algorithm (ForWaRD); the constrained least squares method (CLS); the hybrid spatial and wavelet domains restoration algorithm using the CLS method (CLS-W); the adaptive regularization least squares method (ARLS); and the hybrid spatial and wavelet domains restoration algorithm using the ARLS method (ARLS-W). In the "ARLS" and the "ARLS-W" methods, the corresponding weighting matrices $S$ are computed by using (10) where $\mu=0.5$, and the local variances $\sigma_{i}^{2}$ are estimated from the restored images obtained by the CLS method and the CLS-W method respectively.

Four different image are considered in our test, they are: "Cameraman", "Lenna", "Boat" and "Theater". The PSNRs of the restored images obtained by using different algorithms are summarized in Table 1. In the table, we test several different regularization parameters in each restoration method, and present the best PSNR result of each restoration method. We see from Table 1 that the proposed algorithms perform competitively with ForWaRD. Figure 1 depicts the $256 \times 256$ "Cameraman" image, the obsevered image with the additive noise variance $\sigma^{2}$ be set such that the SNR is $40 \mathrm{~dB}$, and the restoration results for "Cameraman" image using different algorithms. For the spatial reason, others images don't show in here.

The second example is tested by Figueiredo andNowak, ${ }^{3}$ the blurring function is given by

$$
h(i, j)=\frac{1}{1+i^{2}+j^{2}} \quad \text { for } \quad-7 \leq i, j \leq 7 \quad \text { and } \quad h(i, j)=0 \quad \text { otherwise. }
$$

The restoration results are summarized in Table 2. As an example, Figure 2 shows the original image, the observed image and the restored images of "Lenna" using different algorithms. We see from Table 2 and Figure 2 that the proposed algorithms (CLS-W and ARLS-W) perform quite effectively for image restoration.

\begin{tabular}{|c|c|c|c|c|c|c|c|c|c|c|c|c|}
\hline & \multicolumn{3}{|c|}{ Cameraman } & \multicolumn{3}{|c|}{ Lenna } & \multicolumn{3}{|c|}{ Boat } & \multicolumn{3}{|c|}{ Theater } \\
\hline & $30 \mathrm{~dB}$ & $40 \mathrm{~dB}$ & $50 \mathrm{~dB}$ & $30 \mathrm{~dB}$ & $40 \mathrm{~dB}$ & $50 \mathrm{~dB}$ & $30 \mathrm{~dB}$ & $40 \mathrm{~dB}$ & $50 \mathrm{~dB}$ & $30 \mathrm{~dB}$ & $40 \mathrm{~dB}$ & $50 \mathrm{~dB}$ \\
\hline Wiener & 19.02 & 20.83 & 22.92 & 19.24 & 20.99 & 23.12 & 22.24 & 23.28 & 24.93 & 20.31 & 22.37 & 24.39 \\
\hline CLS & 18.99 & 21.07 & 23.45 & 19.45 & 21.49 & 23.99 & 22.73 & 25.56 & 28.76 & 20.00 & 23.17 & 26.40 \\
\hline ARLS & 20.50 & 22.89 & 25.69 & 20.52 & 22.98 & 25.90 & 24.05 & 26.98 & 29.50 & 21.64 & 24.65 & 27.18 \\
\hline ForWaRD & 20.29 & 22.61 & 25.06 & 20.29 & 22.54 & 25.20 & 23.72 & 26.11 & 28.63 & 21.25 & 23.87 & 26.33 \\
\hline CLS-W & 20.43 & 22.79 & 25.49 & 20.45 & 22.91 & 25.77 & 24.03 & 26.67 & 29.76 & 21.90 & 24.62 & 27.47 \\
\hline ARLS-W & 20.84 & 23.33 & 26.02 & 20.71 & 23.09 & 26.04 & 24.40 & 27.16 & 29.88 & 21.93 & 24.85 & 27.42 \\
\hline
\end{tabular}

Table 1. The PSNRs of the restored images by using different algorithms.

\section{CONCLUSION}

In this note, we presented other deblurring schemes in ForWaRD for image restoration. In particular, we studied spatial-domain deblurring methods like constrained least squares restoration and adaptive regularized least squares restoration. Simulation results show that such two-step method is better than traditional image restoration methods. 

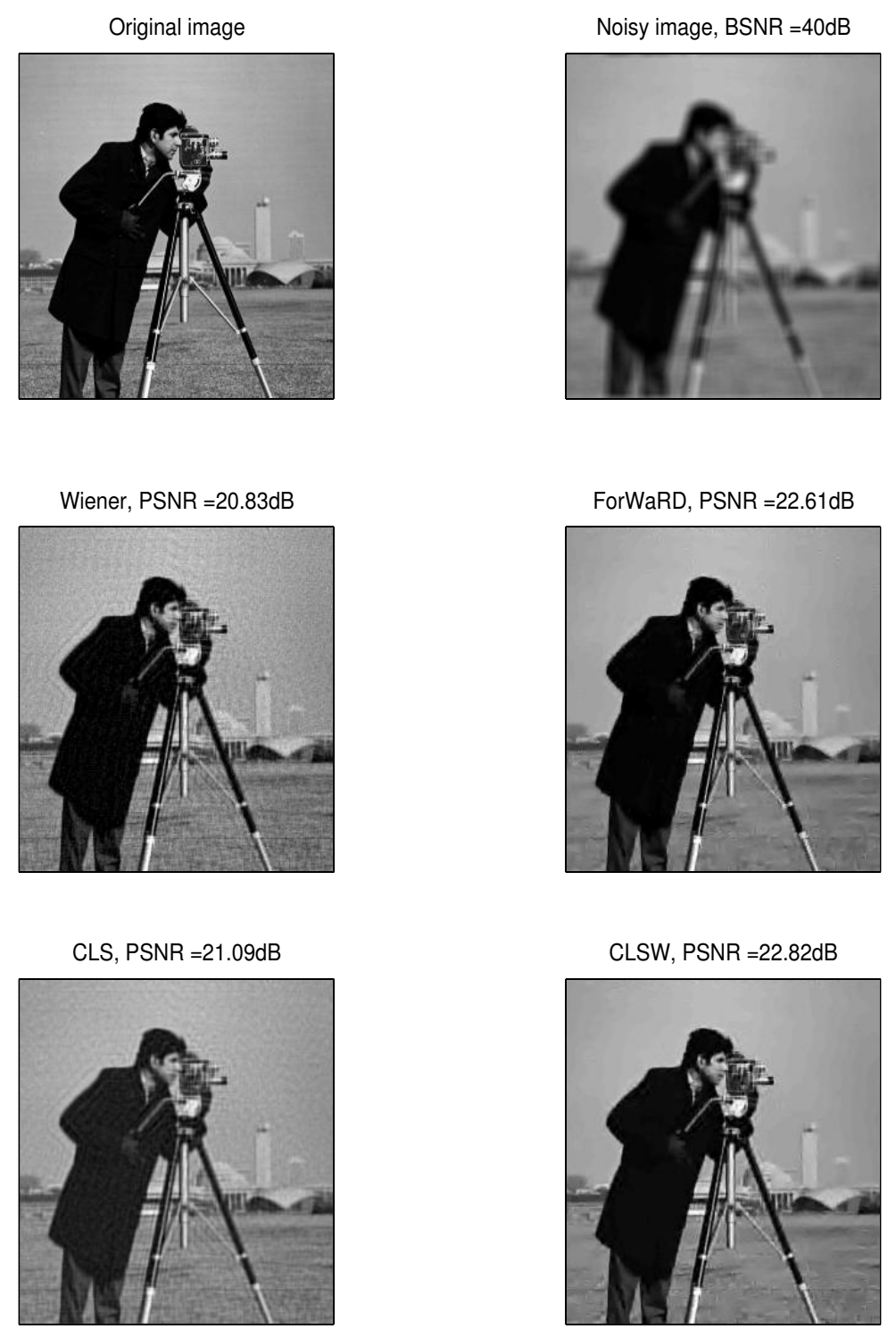

ARLS, PSNR $=22.87 \mathrm{~dB}$

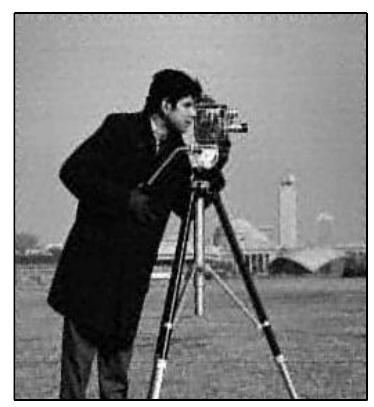

ARLSW, PSNR $=23.33 \mathrm{~dB}$

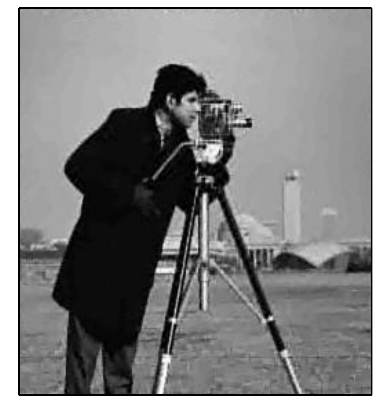

Figure 1. The Original image, the blurred and noisy image, the restored images using the Wiener filter, the ForWaRD with with $\alpha=1.04 \times 10^{-4}$ the CLS with $\alpha=4.00 \times 10^{-4}$, the CLS-W with $\alpha=3.20 \times 10^{-5}$, the ARLS $\alpha=2.50 \times 10^{-2}$ and the ARLS-W $\alpha=2.50 \times 10^{-2}$. 

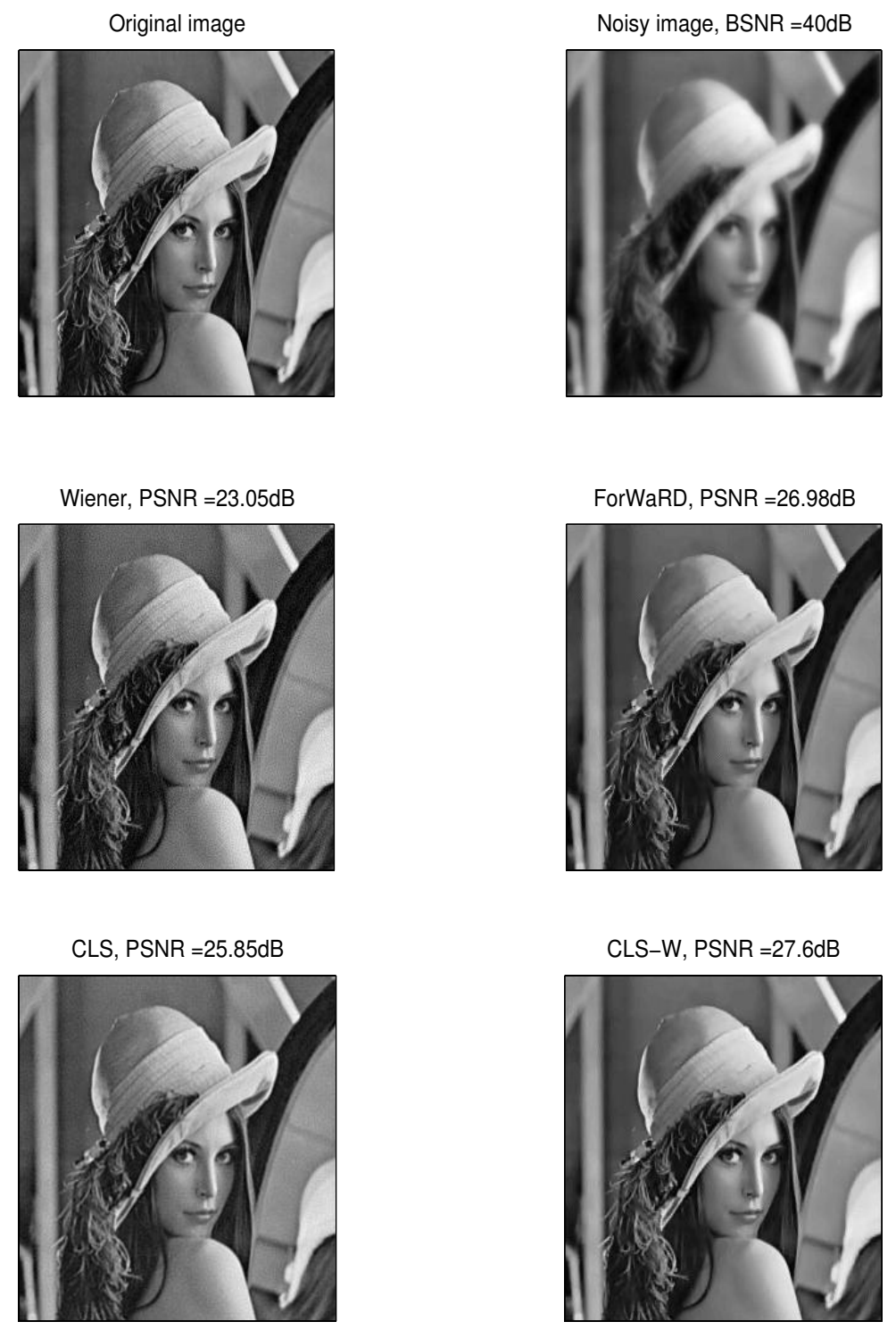

ARLS, PSNR $=27.18 \mathrm{~dB}$

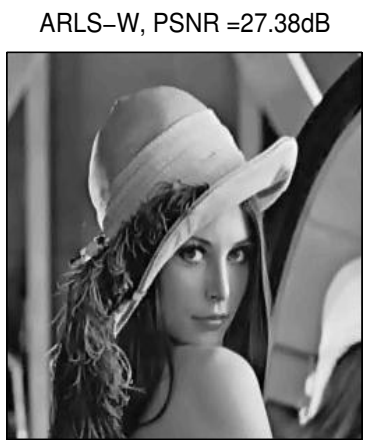

Figure 2. The Original image, the blurred and noisy image, the restored image using the Wiener filter, the ForWaRD with with $\alpha=6.71 \times 10^{-3}$, using the CLS with $\alpha=9.31 \times 10^{-3}$, the CLS-W with $\alpha=1.86 \times 10^{-5}$, the ARLS $\alpha=1.24 \times 10^{-2}$ and the ARLS-W $\alpha=1.24 \times 10^{-2}$. 
Table 2. The PSNRs of the restored images by using different algorithms.

\begin{tabular}{|c|c|c|c|c|c|c|c|c|c|c|c|c|}
\hline & \multicolumn{3}{|c|}{ Cameraman } & \multicolumn{3}{|c|}{ Lenna } & \multicolumn{3}{|c|}{ Boat } & \multicolumn{3}{|c|}{ Theater } \\
\hline & $30 \mathrm{~dB}$ & $40 \mathrm{~dB}$ & $50 \mathrm{~dB}$ & $30 \mathrm{~dB}$ & $40 \mathrm{~dB}$ & $50 \mathrm{~dB}$ & $30 \mathrm{~dB}$ & $40 \mathrm{~dB}$ & $50 \mathrm{~dB}$ & $30 \mathrm{~dB}$ & $40 \mathrm{~dB}$ & $50 \mathrm{~dB}$ \\
\hline Wiener & 15.63 & 21.16 & 28.25 & 15.85 & 23.05 & 28.66 & 17.89 & 23.34 & 30.24 & $\begin{array}{l}17.32 \\
\end{array}$ & 23.20 & 30.05 \\
\hline CLS & 21.54 & 24.88 & 30.01 & 22.58 & 25.85 & 30.57 & 25.33 & 29.04 & 34.37 & 23.38 & 27.55 & 32.83 \\
\hline ARLS & 23.01 & 20.57 & 31.63 & 23.55 & 27.18 & 31.08 & 26.18 & 30.10 & 35.12 & 24.22 & 28.23 & 33.31 \\
\hline ForWaRD & 22.42 & 26.78 & 32.16 & 23.31 & 26.98 & 31.90 & 26.04 & 29.80 & 35.35 & 23.85 & 27.56 & 33.05 \\
\hline CLS-W & 23.37 & 27.12 & 32.39 & 23.91 & 27.60 & 32.13 & 26.83 & 30.83 & 35.74 & 24.79 & 28.77 & 33.55 \\
\hline ARLS-W & 21.08 & 25.58 & 32.58 & 22.17 & 27.38 & 32.20 & 24.77 & 30.66 & 35.69 & 22.16 & 28.47 & 33.69 \\
\hline
\end{tabular}

\section{REFERENCES}

1. N. P. Galatsanos and A. K. Katsaggelos, "Methods for choosing the regularization parameter and estimating the noise variance in image restoration and their relation," IEEE Transactions on Image Processing 1, pp. 322-336, 1992.

2. M. R. Banham and A. K. Katsaggelos, "Spatially adaptive wavelet-based multiscale image restoration," IEEE Transactions on Image Processing 5, pp. 619-634, 1996.

3. M. A. Figueiredo and R. D. Nowak, "An em algorithm for wavelet-based image restoration," IEEE Transactions on Image Processing 12, pp. 906-916, 2003.

4. R. D. Nowak and M. J. Thul, "Wavelet-vaguelette restoration in photon-limited imaging," in Proceedings of the 1998 IEEE International Conference on Acoustics, Speech, and Signal Processing, 5, pp. 2869-2872, 1998.

5. R. Neelamani, H. Choi, and R. Baraniuk, "Forward: Fourier-wavelet regularized deconvolution for illconditioned systems," IEEE Transactions on Signal Processing 52, pp. 418-433, 2004.

6. H. Andrew and B. Hunt, Digital Image Restoration, Prentice-Hall, Englewood Cliffs, NJ, 1977.

7. A. Hillery and R. Chin, "Iterative wiener filters for image restoration," IEEE Transaction on Signal Processing 39, pp. 1892-1899, 1991.

8. D. L. Donoho, "Nonlinear solution of linear inverse problems by wavelet-vaguelette decompositions," $J$. Appl. Comput. Harmon. Anal. 1, pp. 100-115, 1995.

9. R. Gopinath, M. Lang, H. Guo, and J. Odegard, "Enhancement of decompressed images at low bit rates," In SPIE Math. Imagaging: Wavelet Applications in Signal and Image Processing 2303, pp. 366-377, 1994.

10. I. Daubechies, "Orthonorormal bases of compactly supported wavelets," Communications on Pure and Applied Mathematics 41, pp. 909-996, 1988.

11. D. L. Donoho, "Denoising by soft-thresholding," IEEE Trans. Inform. Theory 41, pp. 613-627, 1995.

12. D. L. Donoho and I. M. Johnstone, "Ideal spatial adaptation by wavelet shrinkage," Biometrika 81, pp. 425455, 1994.

13. S. Ghael, A. Sayeed, and R. Baraniuk, "Improved wavelet denoising via empirical wiener filtering," Proc. SPIE. Wavelet Applications in Signal and Image Processing V 3169, pp. 389-399, 1997.

14. M. G. Kang and A. K. Katsaggelos, "General choice of the regularization functional in regularized image restoration," IEEE Transactions on Image Processing 4, pp. 594-602, 1995.

15. J. Biemond and R. Lagendijk, "Regularized iterative image restoration in a weighted hilbert space," in Acoustics, Speech, and Signal Processing, IEEE International Conference on ICASSP '86., 11, pp. 1485$1488,1986$. 